\title{
Agentes ligantes para concretos refratários: sílica coloidal e alumina hidratável ${ }^{*}$
}

\section{(Colloidal silica and hydratable alumina as refractory binder agents)}

\author{
M. R. Ismael, R. Salomão, L. A. Polito, V. C. Pandolfelli \\ Departamento de Engenharia de Materiais, Universidade Federal de S. Carlos \\ Rod. Washington Luiz, km 235, C.P. 676, S. Carlos, SP 13565-905 \\ marinaismael@polvo.ufscar.br,vicpando@power.ufscar.br
}

\begin{abstract}
Resumo
Sílica coloidal e alumina hidratável destacam-se como importantes alternativas à substituição do cimento de aluminato de cálcio como agentes ligantes para concretos refratários aluminosos. Baseando-se nas vantagens e desvantagens destes agentes ligantes isentos de $\mathrm{CaO}$, é razoável supor que a combinação de ambos poderia maximizar os benefícios e reduzir os aspectos negativos observados quando estes são individualmente aplicados. A elevada permeabilidade devido à presença de sílica coloidal poderia reduzir os riscos de explosão durante a secagem das composições ligadas por alumina hidratável. Em paralelo, a porosidade total do sistema contendo sílica coloidal poderia ser diminuída devido à presença de alumina hidratável. Neste trabalho, o desempenho de concretos refratários aluminosos contendo sílica coloidal e alumina hidratável como sistema ligante foram avaliados quanto ao comportamento de mistura, permeabilidade, porosidade aparente, resistência mecânica e perfil de secagem.

Palavras-chave: concretos refratários, agentes ligantes, sílica coloidal, alumina hidratável.
\end{abstract}

\begin{abstract}
Colloidal silica and hydratable alumina can be pointed out as important substitutes to the calcium aluminate cements as calciumfree binders for refractory castables. Based on the advantages and drawbacks of these binders, it is reasonable to consider that their combination could maximize the advantages and reduce the negative aspects observed when they are individually used. The high permeability level generated by the colloidal silica would reduce the risks of explosive spalling of hydratable alumina containing castables during first heat-up. Additionally, the total porosity of the colloidal silica containing systems could be decreased due to the hydration of the HA. In the present work, colloidal silica and hydratable alumina were combined in a new calcium-free binding system. Their rheological behavior, permeability level, apparent porosity, mechanical strength and drying rate profile were compared with the performance of each binder individually.
\end{abstract}

Keywords: refractory castables, binder agents, colloidal silica, hydratable alumina.

\section{INTRODUÇÃOO}

Cimento de aluminato de cálcio (CAC) é o agente ligante mais utilizado em concretos refratários devido à elevada resistência mecânica a verde que proporciona para esses materiais $[1,2]$. No entanto, devido à presença de cálcio em sua composição, o uso de CAC em sistemas contendo microssílica e magnésia é limitado devido à formação de fases de baixo ponto de fusão [2]. Com a finalidade de minimizar esta desvantagem, diferentes agentes ligantes para concretos refratários têm sido desenvolvidos, como a sílica coloidal (SC) e a alumina hidratável (HA) [3-9].

Sílica coloidal é uma dispersão estável de partículas nanométricas de sílica em meio líquido [10]. Quando aplicada em concretos refratários, com o adequado uso de dispersantes e agentes gelificantes, a resistência mecânica é desenvolvida pela formação de uma rede tridimensional

* Prêmio: $50^{\circ}$ Congresso Brasileiro de Cerâmica (2006) entre as partículas devido à gelificação do sol $[2,7]$

Concretos refratários contendo sílica coloidal como agente ligante apresentam menor tempo total de mistura que os tradicionalmente empregados $\mathrm{CAC}$, além de baixa susceptibilidade às condições de cura (tempo e temperatura). Além disso, o mecanismo de consolidação da sílica coloidal não forma fases hidratadas, como o cimento aluminoso, gerando uma estrutura altamente permeável e porosa $[7$, 9]. Este fato é tecnologicamente importante, uma vez que promove a redução do tempo total de processamento e permite que a etapa de secagem seja facilitada, reduzindo o risco de explosão. No entanto, trabalhos anteriores demonstraram que a estrutura altamente porosa formada após a consolidação da sílica coloidal pode ser prejudicial à resistência mecânica e à deformação em elevadas temperaturas [7-9].

Aluminas hidratáveis são aluminas de transição geralmente originadas por meio da calcinação rápida da gibsita $\left(\alpha-\mathrm{Al}_{2} \mathrm{O}_{3} \cdot 3 \mathrm{H}_{2} \mathrm{O}\right)$, resultando principalmente na 
alumina-rho [11-14]. A habilidade ligante deste material deriva da sua capacidade de facilmente se re-hidratar quando em contato com a água. Durante o aquecimento, as fases hidratadas perdem a água quimicamente ligada, originando a forma estável da alumina $\left(\alpha-\mathrm{Al}_{2} \mathrm{O}_{3}\right)$, que em elevadas temperaturas influenciará no desenvolvimento das ligações cerâmicas [11-14]. Quando usadas em substituição ao cimento refratário, as aluminas hidratáveis são geralmente combinadas com microssílica para formar mulita in-situ. Uma vantagem deste agente ligante é o fato de que não requer umidade adicional para desenvolver resistência mecânica. Além disso, ao contrário do que é observado nos concretos refratários contendo $\mathrm{CAC}$, as propriedades dos concretos contendo alumina hidratável são pouco dependentes do tempo de cura. A $20^{\circ} \mathrm{C}$, os materiais rapidamente atingem o máximo de suas propriedades mecânicas. [11-14]. No entanto, não obstante a estas vantagens, a etapa de secagem destes materiais deve ser precisamente controlada, uma vez que os hidratos formados reduzem consideravelmente a permeabilidade dos concretos, aumentando os riscos de explosão durante o aquecimento inicial $\left(200-300{ }^{\circ} \mathrm{C}\right)$ [15]. Soma-se a isso o fato de que, devido à elevada área superficial desse agente ligante $\left(200-300 \mathrm{~m}^{2} / \mathrm{g}\right)$, grandes teores de água e de dispersante são necessários, além de um tempo adicional de mistura, quando comparado aos concretos ligados com CAC. Adicionalmente, a literatura destaca o decréscimo de resistência mecânica que ocorre em temperaturas de secagem intermediárias $\left(300-1000^{\circ} \mathrm{C}\right)$, relacionadas à decomposição das fases hidratadas [11].

Baseando-se nas vantagens e desvantagens destes agentes ligantes isentos de $\mathrm{CaO}$, é razoável supor que a combinação de ambos poderia maximizar os benefícios e reduzir os aspectos negativos observados quando estes são individualmente aplicados. A elevada permeabilidade devido à presença de sílica coloidal poderia reduzir os riscos de explosão durante a secagem das composições ligadas por alumina hidratável. Em paralelo, a porosidade total do sistema contendo sílica coloidal poderia ser diminuída devido à presença de alumina hidratável. Neste trabalho, o desempenho de concretos refratários aluminosos contendo sílica coloidal e alumina hidratável como sistema ligante foi avaliado quanto ao comportamento de mistura, permeabilidade, porosidade aparente, resistência mecânica e perfil de secagem.

\section{MATERIAIS E MÉTODOS}

Foram desenvolvidas três composições de concretos refratários formuladas segundo o modelo de distribuição de partículas de Andreasen [16], com coeficiente $\mathrm{q}=0,21$ : sílica coloidal (SC), alumina hidratável (AH) e o sistema combinado sílica coloidal e alumina hidratável ( $\mathrm{SC}+\mathrm{AH})$. As matériasprimas utilizadas compuseram uma mistura de matriz fina $\left(\mathrm{d}_{\mathrm{p}}<\right.$ $100 \mu \mathrm{m}$ - E-SY Pump 1000, Almatis, EUA) e agregados $\left(\mathrm{d}_{\max }^{\mathrm{p}}\right.$ $=4,75 \mathrm{~mm}$ - alumina eletrofundida branca, Elfusa, Brasil). À formulação SC foi adicionada 7,5\%-p de sílica coloidal (Bindzil, Nalco, $40 \%$ em peso de $\mathrm{SiO}_{2}$ ); à formulação $\mathrm{AH}$, 3,0\%-p de alumina hidratável (Alphabond 300, Almatis, EUA) e 4,5\%-p de água destilada e à composição $\mathrm{SC}+\mathrm{AH}, 7,5 \%$-p de sílica coloidal e 3,0\%-p de alumina hidratável. Ácido cítrico (Labsynth, Brasil) foi utilizado como agente dispersante para as composições $\mathrm{SC}$ e $\mathrm{SC}+\mathrm{AH}$, enquanto que um polietilenoglicol foi empregado no sistema $\mathrm{AH}$. Para as formulações contendo sílica coloidal ( $\mathrm{SC}$ e $\mathrm{SC}+\mathrm{AH})$, sínter de $\mathrm{MgO}\left(\mathrm{d}_{50}=15 \mu \mathrm{m}\right.$; 98\% - p MgO) foi usado como agente gelificante.

A mistura foi feita em um reômetro desenvolvido para concretos sob rotação constante $(44 \mathrm{rpm})$ [17]. Após a homogeneização, as composições foram moldadas na forma de cilindros de $40 \times 40 \mathrm{~mm}$ para os ensaios de secagem, porosidade e resistência mecânica; e $22 \mathrm{~mm}$ de altura por 75 mm de diâmetro para as medidas de permeabilidade. A cura das composições foi realizada em uma câmara climatizada (Vötsch 2020), a $50^{\circ} \mathrm{C}$ durante $24 \mathrm{~h}$, em ambiente insaturado (ao ar) [7]. As amostras para os ensaios de permeabilidade e porosidade foram previamente secas em sílica gel durante $72 \mathrm{~h}$.

Para a determinação da permeabilidade ao ar em temperatura ambiente, as constantes de permeabilidade darciana $\left(\mathrm{k}_{1}\right)$ e não-darciana $\left(\mathrm{k}_{2}\right)$ foram calculadas por meio do ajuste polinomial de pares de dados de pressão imposta e vazão resultante à equação de Forchheimer (A) [18], expressa para fluidos compressíveis como:

$$
\frac{P_{i}^{2}-P_{o}^{2}}{2 P L}=\frac{\mu V_{S}}{k_{1}}+\frac{\rho V_{S}^{2}}{k_{2}}
$$

onde $\mathrm{P}_{\mathrm{i}}$ e $\mathrm{P}_{\mathrm{o}}$ são, respectivamente, a pressão do ar na entrada e saída da amostra; $V_{S}$ é a velocidade do fluido; $L$ é a espessura da amostra; $\mu$ é a viscosidade e $\rho$ a densidade do fluido na temperatura do ensaio (temperatura ambiente).

A porosidade aparente foi obtida pelo método de porosimetria de Arquimedes, utilizando querosene como líquido de imersão (norma ASTM C 20-87). O ensaio de resistência mecânica (compressão diametral - Norma ASTM C 496-90) foi realizado utilizando-se uma máquina de ensaios universal MTS $810 \mathrm{com}$ taxa de aplicação de carga constante $(11 \mathrm{~N} / \mathrm{s})$. Para este ensaio, foram utilizadas amostras secas nas temperaturas de $50{ }^{\circ} \mathrm{C}, 110^{\circ} \mathrm{C}, 350{ }^{\circ} \mathrm{C}$, $600{ }^{\circ} \mathrm{C}, 800{ }^{\circ} \mathrm{C}$ e $1000{ }^{\circ} \mathrm{C}$ com taxa de aquecimento de $3{ }^{\circ} \mathrm{C} / \mathrm{min}$, durante 5 horas.

O ensaio de secagem foi realizado em corpos de provas úmidos (imediatamente após cura), em um equipamento que consiste de uma balança eletrônica ( $400 \pm 0,002 \mathrm{~g})$ acoplada a um forno elétrico [19]. Foram coletadas simultaneamente a perda de massa e a temperatura da superfície da amostra. O aquecimento foi efetuado a $5{ }^{\circ} \mathrm{C} / \min \left(30-600{ }^{\circ} \mathrm{C}\right) \mathrm{com}$ patamar de $10 \mathrm{~min}$ a $600{ }^{\circ} \mathrm{C}$. A perda de massa foi avaliada pelo parâmetro $\mathrm{W}$, que indica a perda de água que ocorre durante o aquecimento em relação a massa final de concreto seco, e sua derivada em relação ao tempo (taxa de secagem), definidos respectivamente nas equações B e C:

$$
W(\%)=100 \times\left(\frac{M_{o}-M}{M_{o}-M_{f}}\right)
$$




$$
\frac{d W}{d t}(\% / \min )=\frac{d}{d t}\left(\frac{M_{0}-M}{M_{0}-M_{f}}\right)
$$

onde $\mathrm{M}$ é a massa instantânea medida no tempo $t_{\mathrm{i}}$, $\mathrm{M}_{\mathrm{o}} \mathrm{a}$ massa inicial e $\mathrm{M}_{\mathrm{f}}$ a massa final.

\section{RESULTADOS E DISCUSSÃO}

\section{Mistura}

A Fig. 1 apresenta o comportamento de mistura para os concretos refratários desenvolvidos contendo diferentes agentes ligantes. $\mathrm{O}$ processo de mistura dos concretos refratários é composto por 3 estágios: mistura a seco, mistura a úmido e ponto de virada e homogeneização final [20].

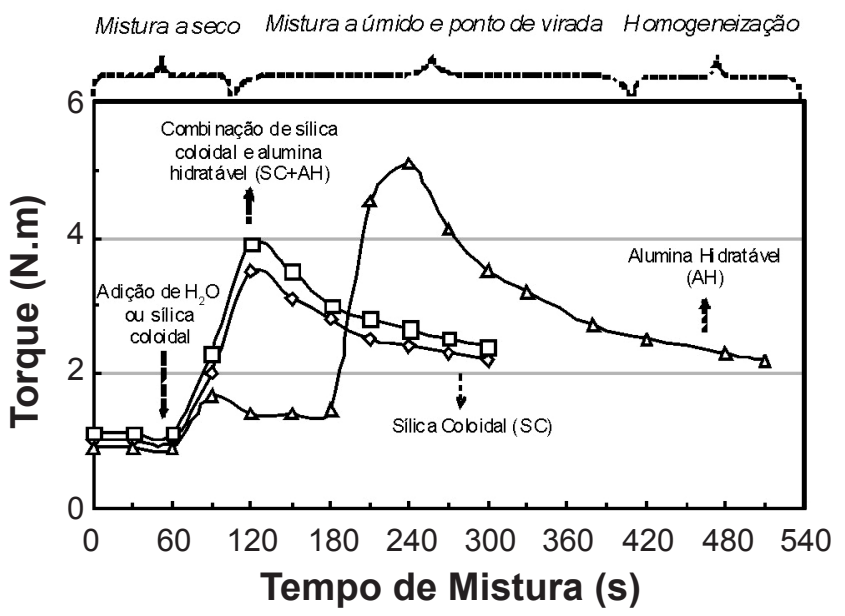

Figura 1: Comportamento de mistura para os sistemas contendo diferentes agentes ligantes.

[Figure 1: Mixing behavior for the refractory castable compositions containing different binder systems.]

A formulação contendo sílica coloidal proporcionou o comportamento de mistura mais favorável, apresentando valores inferiores de torque para alcançar o ponto de virada, além de um menor tempo necessário para a completa mistura. Isto pode ser associado a dois fatores principais [7, 8]: 1) a suspensão de sílica coloidal apresenta maior viscosidade que a água, o que mantém as partículas sólidas mais separadas, facilitando a mistura e 2) a sílica coloidal não promove nenhuma reação química durante esta etapa do processo, como os concretos contendo cimento aluminoso, evitando alterações no $\mathrm{pH}$ e na força iônica.

O tempo adicional de mistura apresentado pela composição AH é uma preocupação considerável em relação ao uso deste agente ligante em concretos refratários. Isto se deve à elevada área superficial da alumina hidratável, o que dificulta o molhamento e a correta atuação do dispersante [11-14]. A adição simultânea de sílica coloidal e alumina hidratável $(\mathrm{SC}+\mathrm{AH})$ originou um comportamento de mistura intermediário entre aqueles observados para cada ligante de forma isolada. $\mathrm{O}$ sistema $\mathrm{SC}+\mathrm{AH}$ apresentou menor tempo total de mistura que a composição $\mathrm{AH}$ e valores de torque ligeiramente superiores àqueles observados para a formulação SC.

\section{Permeabilidade, porosidade e resistência mecânica}

Para análise dos resultados de permeabilidade, porosidade e resistência mecânica das composições $\mathrm{SC}, \mathrm{AH}$ e $\mathrm{SC}+\mathrm{AH}$ (e CAC como referência), apresentados nas Figs. 2 e 3, é necessário entender o mecanismo de consolidação destes agentes ligantes.

Para a composição contendo sílica coloidal, os níveis superiores de permeabilidade e porosidade e a resistência mecânica inferior (abaixo de $350{ }^{\circ} \mathrm{C}$ ) estão associados à estrutura de elevada porosidade gerada devido ao mecanismo de gelificação, que ocorre quando os grupos silanol (-Si-OH-) presentes na superfície das partículas reagem entre si e se condensam [10]. Essa reação resulta em uma ligação silano (-Si-O-Si-) que une as partículas e as torna hidrofóbicas. Novas partículas se unem a esse aglomerado e uma rede tridimensional é formada [10].

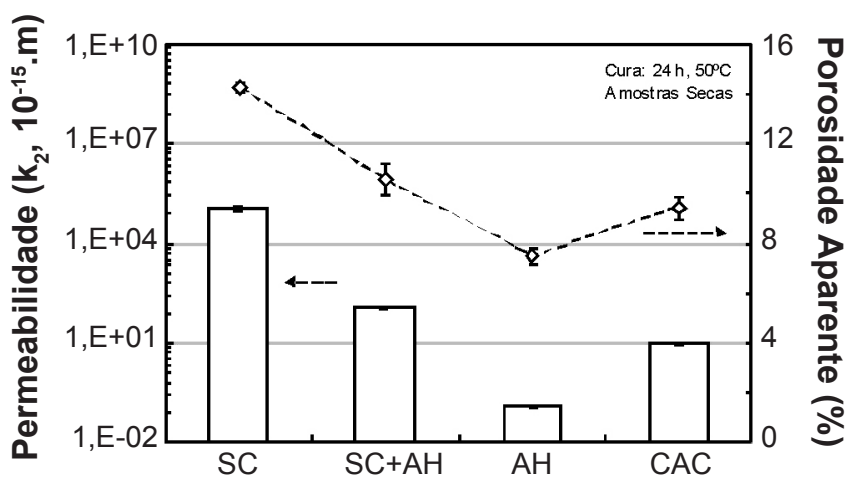

Figura 2: Permeabilidade e porosidade aparente para os sistemas contendo diferentes agentes ligantes.

[Figure 2: Permeability and porosity levels for the refractory castable compositions containing different binder systems.]

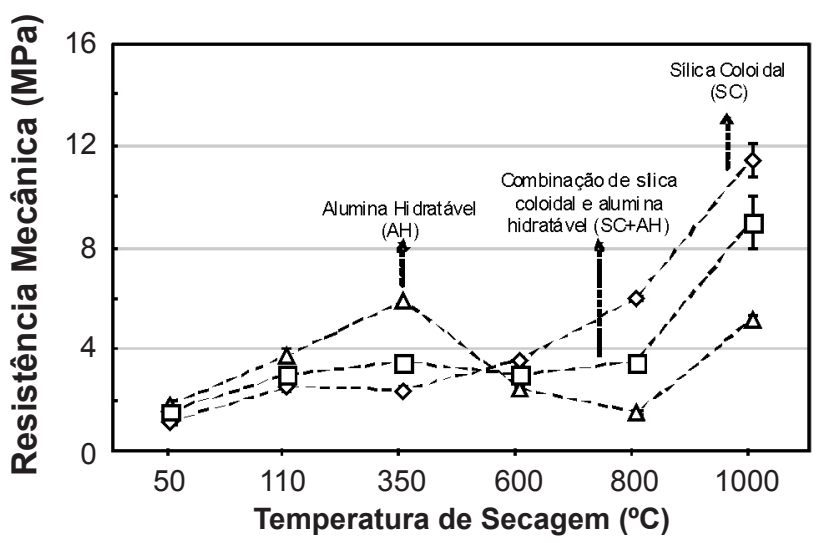

Figura 3: Resistência mecânica em função da temperatura de secagem para os sistemas contendo diferentes agentes ligantes. [Figure 3: Mechanical strength as a function of drying temperature for refractory castable compositions containing different binder systems.] 
Para o sistema ligado com alumina hidratável, o mecanismo de consolidação é baseado na cristalização de um gel nanométrico, capaz de fechar os poros e os canais permeáveis da estrutura (Fig. 2), promovendo elevada resistência mecânica inicial (Fig. 3) [11-15].

Os resultados da Fig. 2 indicam que o sistema combinado $(\mathrm{SC}+\mathrm{AH})$ gerou uma estrutura com características intermediárias: não tão porosa e permeável como a composição contendo sílica coloidal, nem tão empacotada como a ligada por alumina hidratável - similar ao nível geralmente apresentado pelas composições contendo CAC. A Fig. 3 indica que a resistência mecânica do sistema contendo ambos agentes ligantes também apresentou um comportamento intermediário. Em baixas temperaturas de secagem (abaixo de $350{ }^{\circ} \mathrm{C}$ ), a alumina hidratável, devido a sua elevada área superficial, potencializou o mecanismo de gelificação do sol: o aumento da quantidade de matriz no sistema originou uma maior área para ancoramento da rede tridimensional, resultando em uma estrutura mais coesa. Um efeito similar é constatado na literatura quando há a introdução de microssílica em sistemas ligados por sílica coloidal [9]. Em temperaturas de secagem superiores a $350{ }^{\circ} \mathrm{C}$, o decréscimo de resistência mecânica apresentado pelo sistema $\mathrm{AH}$ foi minimizado com a presença da sílica coloidal.

\section{Perfil de secagem}

O perfil de secagem de concretos refratários é geralmente dividido em três estágios [19]. O primeiro consiste na evaporação da água livre (da temperatura ambiente até $100{ }^{\circ} \mathrm{C}$ ). Acima de $100{ }^{\circ} \mathrm{C}$, até $200-300{ }^{\circ} \mathrm{C}$, dependendo do ligante utilizado, o estágio de ebulição é verificado. O terceiro estágio relacionase à decomposição das fases hidratadas formadas devido à natureza do agente ligante presente (acima de $250-300^{\circ} \mathrm{C}$ ).

Recentes trabalhos demonstraram que para concretos contendo sílica coloidal ou alumina hidratável como agente ligante, a etapa de cura deve ser realizada em ambiente

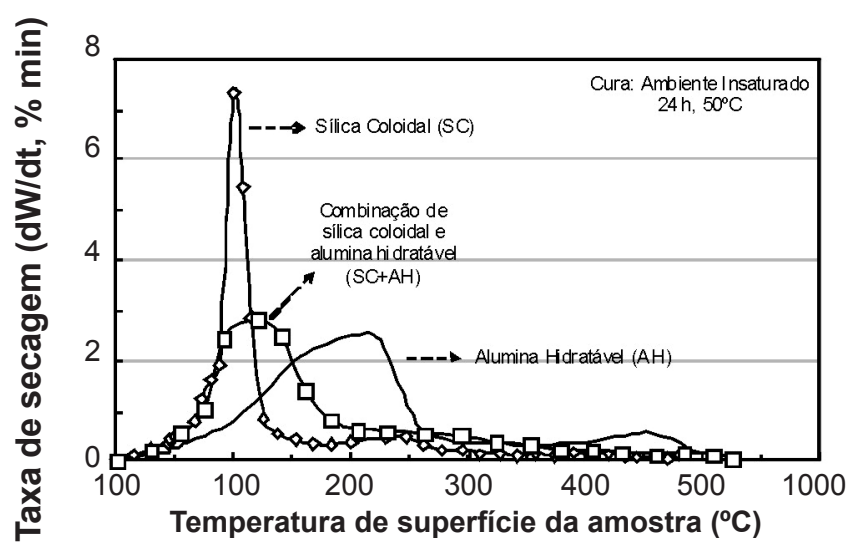

Figura 4: Taxa de secagem $\left(5^{\circ} \mathrm{C} / \mathrm{min}\right)$ em função da temperatura da superfície da amostra para os sistemas contendo diferentes agentes ligantes.

[Figure 4: Drying rate $\left(5^{\circ} \mathrm{C} / \mathrm{min}\right)$ as a function of temperature at the surface of the samples of refractory castable compositions containing different binder systems.] não saturado de vapor de água, isto é, ao ar [7]. Assim, a remoção da água fisicamente ligada durante a cura (efeito da pré-secagem) explica a ausência do pico de evaporação nessas composições (Fig. 4).

Verifica-se que o concreto ligado por sílica coloidal apresentou o perfil de secagem mais adequado: elevada velocidade e baixa temperatura no final do estágio de ebulição (abaixo de $150^{\circ} \mathrm{C}$ ). Este fato é relacionado tanto à estrutura altamente porosa e permeável que é formada com a adição deste ligante, como devido a não existência de fases hidratadas, o que também reduz os riscos de pressurização durante esta etapa do processo. A maior dificuldade de secagem apresentada pela composição $\mathrm{AH}$ relaciona-se à capacidade do gel oriundo do mecanismo de consolidação em preencher os canais permeáveis presentes na estrutura desses concretos [15]. Além disso, o final da ebulição desse sistema ocorreu em elevada temperatura, o que pode indicar grande risco de explosão. O pico de perda de massa observado entre 400 e $500{ }^{\circ} \mathrm{C}$ para essa formulação é devido à desidratação da boemita [15].

O sistema combinado $(\mathrm{SC}+\mathrm{AH})$ apresentou uma taxa de secagem menor do que a observada para a composição SC, no entanto mais rápida do que a apresentada para $\mathrm{AH}$. Esse resultado pode ser relacionado ao nível de permeabilidade e porosidade desse sistema (Fig. 2). A ausência da decomposição do pico de boemita na formulação $\mathrm{SC}+\mathrm{AH}$ indica que, apesar do aumento no empacotamento das partículas, a hidratação da alumina de transição foi significativamente reduzida. Este aspecto ainda não foi totalmente compreendido, no entanto, o interesse tecnológico nesse sistema sugere que este deve ser explorado para obter concretos com maior resistência mecânica e menor nível de porosidade.

\section{CONCLUSÕES}

Concretos ligados por sílica coloidal e alumina hidratável podem ser processados e curados nas mesmas condições (ambiente insaturado, curto tempo de cura). Enquanto que sistemas contendo sílica coloidal apresentam mistura e secagem facilitada, composições ligadas por alumina hidratável permitem o desenvolvimento de elevada resistência mecânica inicial. Baseando-se nesses fatos, o presente trabalho avaliou a hipótese de que a combinação de sílica coloidal e alumina hidratável como um sistema ligante para concretos refratários poderia trazer importantes vantagens para o processamento e propriedades desses materiais.

Comparando-se com os resultados obtidos para os concretos contendo alumina hidratável, o sistema combinado apresentou menor tempo total de mistura e reduzido risco de explosão durante a etapa de secagem. Além disso, essa composição proporcionou menor sensibilidade à resistência mecânica em temperaturas intermediárias $\left(350-800{ }^{\circ} \mathrm{C}\right)$. Considerando-se a formulação contendo sílica coloidal, o sistema combinado gerou uma redução na porosidade aliada a um aumento de resistência mecânica em temperaturas inferiores a $350{ }^{\circ} \mathrm{C}$. 


\section{AGRADECIMENTOS}

A CAPES, FAPESP e Magnesita S.A. pelo fomento à pesquisa e a Almatis e Elfusa Geral de Eletrofusão Ltda pelas matérias-primas fornecidas.

\section{REFERÊNCIAS}

[1] W. E. Lee, W. Vieira, S. Zhang, K. Ghanbari Ahari, H. Sarpoolaky, C. Parr, Castable refractory concretes, Int. Mater. Rev. 46, 3 (2001) 145.

[2] S. Banerjee, Monolithic Refractories - a comprehensive handbook, World Scientific/The Am. Ceram. Soc., Singapore (1988) 111.

[3] S. Banerjee, Recent developments in monolithic refractories, Am. Ceram. Soc. Bull. 77, 10 (1998) 59.

[4] R. Racher, Improved wokability of calcia free alumina binder Alphabond for non-cement castables, $9^{\text {th }}$ Biennial Worldwide Congress on Refractories, Orlando, USA (November 8-11, 2005).

[5] S. Banerjee, Versality of gel-bond castable/pumpable refractories, Refractories Appl. News 6, 1 (2001) 1.

[6] S. Banerjee, et al. Composition and method for manufacturing steel-containment equipment, Patent $\mathrm{n}$. 5147830 (Sep. 15, 1992).

[7] M. R. Ismael, R. D. Anjos, R. Salomão, V. C. Pandolfelli, Colloidal silica as a nanostructured binder for refractory castables, Refractories Application and News, (2006) aceito.

[8] M. R. Ismael, F. T. Ramal Jr., V. C. Pandolfelli, Sol de sílica como agente ligante para concretos refratários, Cerâmica 52, 321 (2006) 82.

[9] M. R. Ismael, R. Salomão, V. C. Pandolfelli, Otimização do uso de sol de sílica como agente ligante para concretos refratários, Cerâmica 52, 321 (2006) 92 .
[10] R. K. Iler, The chemistry of silica: solubility, polymerization, colloid and surface properties, and biochemistry, Wiley, New York (1979).

[11] Y. Hongo, $\rho$-alumina bonded castable refractories, Taikabutsu Overseas 9, 1 (1988) 35.

[12] W. Ma, P. W. Brown, Mechanisms of reaction of hydratable aluminas, J. Am. Ceram. Soc. 82, 2 (1999) 453.

[13] W. Mista, J. Wrzyszcz, Rehydration of transition aluminas obtained by flash calcination of gibbsite, Thermochimica Acta 331 (1999) 67.

[14] M.W. Vance, J. Moody, Use of hydratable alumina binders in refractory compositions and related applications, 97 $7^{\text {th }}$ ACERS Annual Meeting and Exposition, Cincinnati, Ohio, USA (May 1, 1995).

[15] F. A. Cardoso, M. D. M. Innocentini, M. F. S. Miranda, F. A. O. Valenzuela, V. C. Pandolfelli, Drying behaviour of hydratable alumina-bonded refractory castables, J. Eur. Ceram. Soc. 24, 4 (2004) 797.

[16] I. R. Oliveira, A. R. Studart, R. G. Pileggi, V. C. Pandolfelli, Dispersão e empacotamento de partículas - princípios e aplicações em processamento cerâmico, Fazendo Arte Editorial, S. Paulo, Brasil (2000) 119.

[17] R. G. Pileggi, V. C. Pandolfelli, A. E. Paiva, J. Gallo, Novel rheometer for refractory castables, Am. Ceram. Soc. Bull. 79, 1 (2000) 54.

[18] M. D. M. Innocentini, A. R. F. Pardo, V. R. Salvini, V. C. Pandolfelli, How accurate is Darcy's law for refractories, Am. Ceram. Soc. Bull. 78, 11 (1999) 64.

[19] M. D. M. Innocentini, F. A. Cardoso, M. M. Akiyoshi, V. C. Pandolfelli, Drying stages during the heat-up of highalumina, ultra low cement refractory castables, J. Am. Ceram. Soc. 86, 7 (2003) 1146.

[20] R. G. Pileggi, A. R. Studart, V. C. Pandolfelli, et al., How mixing affects the rheology of refractory castables Part 1, Am. Ceram. Soc. Bull. , 6 (2001) 27.

(Rec. 29/04/2006, Ac. 29/07/2006) 